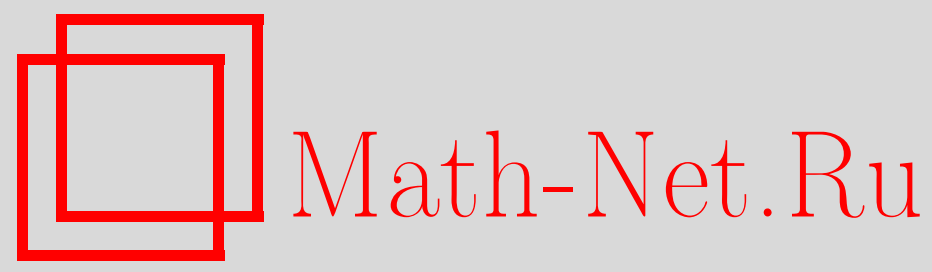

А. Л. Лукашов, Неравенство типа Бернштейна для производных рациональных функций на двух отрезках, Матем. заметки, 1999, том 66, выпуск 4, 508-514

DOI: https://doi.org/10.4213/mzm1194

Использование Общероссийского математического портала Math-Net.Ru подразумевает, что вы прочитали и согласны с пользовательским соглашением http://www.mathnet.ru/rus/agreement

Параметры загрузки:

IP : 54.224 .60 .19

26 апреля 2023 г., $13: 47: 30$

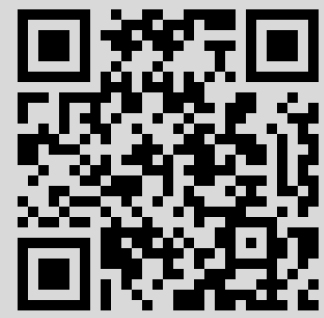


том 66 выпУск 4 оКТяБРь 1999

УДК 517.518.86

\section{НЕРАВЕНСТВО ТИПА БЕРНШТЕЙНА ДЛЯ ПРОИЗВОДНЫХ РАЦИОНАЛЬНЫХ ФУНКЦИЙ НА ДВУХ ОТРЕЗКАХ}

\section{А. Л. Лукашов}

В работе получено неравенство для производных рациональных функций с фиксированньм знаменателем, обобщающее неравенство В.С. Виденского на случай двух отрезков. Для доказательства его асимптотической точности используется одно новое представление дробей Ахиезера-Золотарёва, наименее уклоняющихся от 0 на двух отрезках.

Библиографоия: 15 названий.

1. Введение. Пусть $A_{n}=\left\{a_{1}, \ldots, a_{n}\right\}$-строка обратных величин полюсов, $1 / a_{i} \in$ $\mathbb{C} \backslash E, i=1, \ldots, n, E=[-1, a] \cup[b, 1],-1<a<b<1$, причем для $\operatorname{Im} a_{i}>0$ справедливо равенство $a_{i+1}=\bar{a}_{i}$. Через $\mathscr{R}\left(A_{n}\right)$ будем обозначать класс рациональных функций вида

$$
\frac{x^{n}+b_{1} x^{n-1}+\cdots+b_{n}}{\prod_{i=1}^{n}\left(1-a_{i} x\right)}, \quad b_{i} \in \mathbb{R} .
$$

Основная цель данной работы - получить асимптотически точную оценку производных типа неравенства Бернштейна дроби $R_{n}(x)$, если известна норма $\left\|R_{n}\right\|_{C(E)}$. Для определенности будем считать, что $a_{1}=\cdots=a_{\varkappa}=0$ и $a_{i} \neq 0$ для $i=\varkappa+1, \ldots, n$, $\varkappa>0$. Получен следующий результат.

ТЕОрема 1. Пусть $R_{n}(x)$ - рациональная функция описанного выше вида, причем $A_{n} \subset\{z:|z|<1\}$. Кроме того, предположим, что все действительные нули функиии $R_{n}(x)$ удовлетворяют неравенству $|x|<\min _{\varkappa \leqslant l \leqslant n}\left|1 / a_{l}\right|$. Тогда для $x \in(-1, a)$

$$
\left|R_{n}^{\prime}(x)\right| \leqslant \frac{\left\|R_{n}\right\|_{C(E)}}{\sqrt{1-x^{2}}} \sqrt{\frac{x-b}{x-a}} \sum_{j=1}^{n}\left|\frac{\sqrt{1-a_{j}^{2}}}{1-a_{j} x} \sqrt{\frac{1-a_{j} a}{1-a_{j} b}}\right|
$$

a для $x \in(b, 1)$

$$
\left|R_{n}^{\prime}(x)\right| \leqslant \frac{\left\|R_{n}\right\|_{C(E)}}{\sqrt{1-x^{2}}} \sqrt{\frac{x-a}{x-b}} \sum_{j=1}^{n}\left|\frac{\sqrt{1-a_{j}^{2}}}{1-a_{j} x} \sqrt{\frac{1-a_{j} b}{1-a_{j} a}}\right| .
$$

Работа выполнена при финансовой поддержке Российского фонда фундаментальных исследований, грант № 99-01-01120. 
Отметим, что при $a_{j}=0, j=1, \ldots, n$, утверждение теоремы совпадает с результатом П.Б. Борвейна [1] (см. также [2]), а при $a=b$ неравенства (1) и (2) перейдут в неравенство В. С. Виденского [3] (см. также [4], [5]). Для $E=\{z:|z|=1\}$ и $E$, являющегося простой замкнутой спрямляемой кривой, ограничивающей односвязную область, аналогичные утверждения получены А. А. Пекарским [6] (см. также [7]). Весьма общее неравенство несколько иного типа для оценки производных рациональных функций получено В.И. Данченко [8]. Хорошим обзором результатов по этой тематике и связанным с ней задачам служат также [9, гл. 10] и [10]. Предварительньй вариант данной статьи был изложен в [11].

2. Вспомогательные утверждения. В дальнейшем будем использовать следующие обозначения для эллиптических функций Якоби:

$$
K=K(k)=\int_{0}^{1} \frac{d u}{\sqrt{\left(1-u^{2}\right)\left(1-k^{2} u^{2}\right)}}
$$

- полный эллиптический интеграл первого рода, соответствующий модулю $k, 0<k<1$; $K^{\prime}=K\left(k^{\prime}\right)$, где $k^{\prime}=\sqrt{1-k^{2}} ; H(u), \Theta(u), H_{1}(u), \Theta_{1}(u)$ - тэта-функции в обозначениях Якоби с параметром $h=\exp \left(-\pi K^{\prime} / K\right) ; \operatorname{sn} u=\operatorname{sn}(u ; k), \operatorname{cn} u=\operatorname{cn}(u ; k), \operatorname{dn} u=\operatorname{dn}(u ; k)-$ функции Якоби с параметром $k$.

Назовем строку $A_{n}$ регулярной относительно $E$, если величина

$$
\varphi\left(A_{n}, k, a\right)=\frac{1}{K} \int_{0}^{\sqrt{(1-a) / 2}} \sum_{l=1}^{n} \frac{\sqrt{\left(1+a_{l}\right)\left(1-a a_{l}\right)} d u}{\sqrt{\left(1-a a_{l}-\left(1+a_{l}\right) u^{2}\right)\left(1-a a_{l}-k^{2}\left(1+a_{l}\right) u^{2}\right)}}
$$

принимает целое значение при $k^{2}=2(b-a) /((1-a)(1+b))$. Задачей Чебъиева-Мар$\kappa о в а$ для $E$ называют проблему нахождения функции $R \in \mathscr{R}\left(A_{n}\right)$, доставляющей минимум в

$$
\|R\|_{C(E)} \rightarrow \min _{R \in \mathscr{R}\left(A_{n}\right)} .
$$

Для $E=[-1,1]$ задача была решения П. Л. Чебьшевым и, более полно, А. А. Марковым. Для $E=[-1, a] \cup[b, 1]$ Н.И. Ахиезер [12] решил ряд задач, тесно связанных с (3), но в явном виде (3) не рассматривал. Первое явное решение в одном частном случае в связи с задачами электротехники содержится в [13], где отмечалось, что оно фактически совпадает с дробями Е. И. Золотарёва, поэтому далее соответствующие функции будут назьваться функииями Ахиезера-Золотарёва.

Теорема 2. Пусть $\varphi\left(A_{n}, k, a\right)=m \in \mathbb{N}$. Тогда рациональная функиия $R\left(A_{n}, E, x\right)$, решающая задачу (3), может быть представлена в виде

$$
R\left(A_{n}, E, x\right)=M_{n}\left(\prod_{l=1}^{n} \frac{H\left(u-\rho_{l}\right)}{H\left(u+\rho_{l}\right)}+\prod_{l=1}^{n} \frac{H\left(u+\rho_{l}\right)}{H\left(u-\rho_{l}\right)}\right)
$$

$2 \partial e$

$$
\begin{gathered}
x=\frac{\operatorname{sn}^{2} u \mathrm{cn}^{2} \rho_{0}+\mathrm{cn}^{2} u \mathrm{sn}^{2} \rho_{0}}{\mathrm{sn}^{2} u-\mathrm{sn}^{2} \rho_{0}} \\
\operatorname{sn}^{2} \rho_{l}=\frac{(1-a)\left(1+a_{l}\right)}{2\left(1-a a_{l}\right)}, \quad l=0,1, \ldots, n ; \quad-K^{\prime}<\operatorname{Im} \rho_{l}<K^{\prime}, \quad l=1, \ldots, n ; \\
-K<\operatorname{Re} \rho_{l}<0, \quad l=0,1, \ldots, n ; \quad \operatorname{Im} a_{l}>0 \Longrightarrow \rho_{l}=\bar{\rho}_{l+1} ; \\
M_{n}=\frac{\varepsilon}{\prod_{l=\varkappa+1}^{n} a_{l}} \prod_{l=\varkappa+1}^{n} \frac{H\left(\rho_{0}+\rho_{l}\right)}{H\left(\rho_{0}-\rho_{l}\right)}\left(\frac{\Theta^{2}(0) \Theta_{1}^{2}(0)}{2 \Theta_{1}^{2}\left(\rho_{0}\right) \Theta^{2}\left(\rho_{0}\right)}\right)^{\varkappa}, \quad \varepsilon= \pm 1 ;
\end{gathered}
$$


либо в виде

$$
\begin{aligned}
R\left(A_{n}, E, x\right)= & M_{n}(-1)^{n-n_{3}-n_{2}} \\
& \times \cos \int_{-1}^{x} \sum_{l=1}^{n} \frac{\left(x-c_{l}\right) \sqrt{\left(1-a_{l}^{2}\right)\left(1-a_{l} a\right)\left(1-a_{l} b\right)}}{\left(1-a_{l} x\right)\left(1-a_{l} c_{l}\right) \sqrt{\left(1-x^{2}\right)(x-a)(x-b)}} d x,
\end{aligned}
$$

где $M_{n}$ определяется из равенства $(5)$, а

$$
c_{l}=\frac{\sqrt{1-a_{l} a} \Theta^{\prime}\left(\rho_{l}\right) \sqrt{(1-a)(1+b)}-a \Theta\left(\rho_{l}\right) \sqrt{\left(1-a_{l}^{2}\right)\left(1-a_{l} b\right)}}{a_{l} \sqrt{1-a_{l} a} \Theta^{\prime}\left(\rho_{l}\right) \sqrt{(1-a)(1+b)}-\Theta\left(\rho_{l}\right) \sqrt{\left(1-a_{l}^{2}\right)\left(1-a_{l} b\right)}}, \quad l=1, \ldots, n
$$

причем $a<c_{l}<b$ для $\operatorname{Im} a_{l}=0 ; c_{l}$ лежит на дуге окружности, проходящей через $a, b u 1 / a_{l}$, ограниченной точками $a u b$ не содержащей $1 / a_{l}$, при $\operatorname{Im} a_{l}>0$, $l=1, \ldots, n\left(\right.$ в әтом случае $\left.c_{l+1}=\bar{c}_{l}\right)$.

Подробное доказательство теоремы содержится в [11]. При этом формулы (4)-(7) доказьваются вполне аналогично соответствующим утверждениям работы [12]. Ради полноты рассуждений приведем лишь доказательство геометрической характеристики числа $c_{l}$.

ЛЕмма 1. Для всех $\rho \in \mathbb{C}$ справедливо неравенство

$$
\operatorname{Re} \frac{k^{2} \operatorname{sn} \rho \operatorname{cn} \rho}{\operatorname{dn} \rho}<\operatorname{Re} \frac{\Theta^{\prime}(\rho)}{\Theta(\rho)}<0 .
$$

ДокАЗАТЕЛЬСтво. По формуле из [14, гл. 21, пример 15] имеем

$$
\begin{gathered}
\frac{\Theta^{\prime}(\rho)}{\Theta(\rho)}+\frac{\Theta^{\prime}(\bar{\rho})}{\Theta(\bar{\rho})}=\frac{2 \pi}{K} \sum_{n=1}^{\infty} h^{2 n-1} \operatorname{Re} \frac{\sin (\pi \rho / K)}{1-2 h^{2 n-1} \cos (\pi \rho / K)+h^{4 n-2}} \\
\frac{\Theta_{1}^{\prime}(\rho)}{\Theta_{1}(\rho)}+\frac{\Theta_{1}^{\prime}(\bar{\rho})}{\Theta_{1}(\bar{\rho})}=-\frac{2 \pi}{K} \sum_{n=1}^{\infty} h^{2 n-1} \operatorname{Re} \frac{\sin (\pi \rho / K)}{1+2 h^{2 n-1} \cos (\pi \rho / K)+h^{4 n-2}}
\end{gathered}
$$

Поскольку

$$
\begin{aligned}
& \operatorname{Re} \frac{\sin (\pi \rho / K)}{1 \pm 2 h^{2 n-1} \cos (\pi \rho / K)+h^{4 n-2}} \\
& \quad=\frac{\sin (\pi \operatorname{Re} \rho / K) \operatorname{ch}(\pi \operatorname{Im} \rho / K)\left(1+h^{4 n-2}\right) \pm 4 h^{2 n-1} \sin (\pi \operatorname{Re} \rho / K) \cos (\pi \operatorname{Re} \rho / K)}{\left|1 \pm 2 h^{2 n-1} \cos (\pi \rho / K)+h^{4 n-2}\right|^{2}}<0
\end{aligned}
$$

при $-K<\operatorname{Re} \rho<0$, справедливы неравенства

$$
\operatorname{Re} \frac{\Theta^{\prime}(\rho)}{\Theta(\rho)}<0, \quad \operatorname{Re} \frac{\Theta_{1}^{\prime}(\rho)}{\Theta_{1}(\rho)}>0 .
$$

Учитьвая представление $\Theta_{1}(\rho)=\operatorname{dn} \rho \Theta(\rho) / \sqrt{k^{\prime}}$, получаем утверждение леммы.

ЗАмечаниЕ 1. Для $-K<\rho<0$ неравенство (8) было доказано в работе [12,c. 1184]. 
ДоКАЗАТЕЛЬСТВо ТЕОРЕмЫ 2. Из неравенства (8) получаем, что

$$
0>\operatorname{Re} \frac{\Theta^{\prime}\left(\rho_{l}\right)}{\Theta\left(\rho_{l}\right)}>-\operatorname{Re} \frac{b-a}{\sqrt{(1-a)(1+b)}} \sqrt{\frac{1-a_{l}^{2}}{\left(1-a_{l} a\right)\left(1-a_{l} b\right)}}, \quad l=1, \ldots, n .
$$

Для $\operatorname{Im} a_{l}=0$ из (8) по теореме Лагранжа получаем, что

$$
\begin{aligned}
& \frac{(x-a) \sqrt{\left(1-a_{l}^{2}\right)\left(1-a_{l} b\right)}}{\left(1-a_{l} x\right) \sqrt{1-a_{l} a}}+\frac{\Theta^{\prime}\left(\rho_{l}\right)}{\Theta\left(\rho_{l}\right)} \sqrt{(1-a)(1+b)} \\
& =\frac{\left(x-c_{l}\right) \sqrt{\left(1-a_{l}^{2}\right)\left(1-a_{l} a\right)\left(1-a_{l} b\right)}}{\left(1-a_{l} x\right)\left(1-a_{l} c_{l}\right)},
\end{aligned}
$$

где $a<c_{l}<b$. Если же $\operatorname{Im} a_{l} \neq 0$, то, записав $(7)$ и используя $(8)$, найдем, что

$$
\begin{aligned}
\operatorname{Re} \frac{(x-a) \sqrt{\left(1-a_{l}^{2}\right)\left(1-a_{l} b\right)}}{\left(1-a_{l} x\right) \sqrt{1-a_{l} a}}>\operatorname{Re} \frac{\left(x-c_{l}\right) \sqrt{\left(1-a_{l} a\right)\left(1-a_{l} b\right)\left(1-a_{l}^{2}\right)}}{\left(1-a_{l} x\right)\left(1-a_{l} c_{l}\right)} \\
>\operatorname{Re} \frac{(x-b) \sqrt{\left(1-a_{l}^{2}\right)\left(1-a_{l} a\right)\left(1-a_{l} b\right)}}{\left(1-a_{l} x\right)\left(1-a_{l} b\right)} .
\end{aligned}
$$

Зафиксируем $x \in \mathbb{C} \backslash \bigcup_{l=\varkappa+1}^{n}\left\{1 / a_{l}\right\}$ и обозначим

$$
z_{l, x}=\frac{\sqrt{\left(1-a_{l} a\right)\left(1-a_{l} b\right)\left(1-a_{l}^{2}\right)}}{1-a_{l} x} .
$$

Тогда неравенство (9) означает, что точка $\left(x-c_{l}\right) z_{l, x} /\left(1-a_{l} c_{l}\right)$ лежит в вертикальной полосе, ограниченной прямыми, проходящими через точки $(x-a) z_{l, x} /\left(1-a_{l} a\right)$ и $(x-b) z_{l, x} /\left(1-a_{l} b\right)$. Применяя далее дробно-линейное отображение

$$
w(z)=\frac{z_{l, x} x-z}{z_{l, x}-a_{l} z}
$$

получим, что точка $c_{l}$ лежит между окружностями, касаюшимися друг друга в точке $1 / a_{l}$ и проходящими через точки $a$ и $b$ соответственно ортогонально окружности, проходящей через точки

$$
\frac{z_{l, x} x-a}{z_{l, x}-a_{l} a}, \frac{1}{a_{l}}, \frac{z_{l, x} x-b}{z_{l, x}-a_{l} b} .
$$

Так как $c_{l}$ от $x$ не зависит, а $x$ произвольно, то $c_{l}$ должно принадлежать геометрическому месту точек, находящихся между любыми двумя касающимися друг друга в точке $1 / a_{l}$ окружностями, проходящими через точки $a$ и $b$ соответственно. Применяя отображение

$$
w_{1}=\frac{w\left(1-a_{l} b-a_{l} a\right)-a_{l} a b}{1-a_{l} w},
$$

получим, что точка $w_{1}\left(c_{l}\right)$ должна принадлежать геометрическому месту точек, находящихся между любыми двумя параллельными прямыми, проходящими через $a$ и $b$, т.е. интервалу $(a, b)$. После применения обратного отображения

$$
w=\frac{w_{1}-a_{l} a b}{w_{1} a_{l}+1-a_{l} a-a_{l} b}
$$

получим требуемое. 
ЗАмЕчаниЕ 2. Другая форма представления (4) приведена в [15].

ЛЕмма 2. Пусть $E \subset K$ - произвольное бесконечное компактное множество $u \zeta \in \mathbb{R}$. Предположим, что функиия $R_{n} \in \mathscr{R}\left(A_{n}\right)$ такая, что

$$
\frac{\left|R_{n}^{\prime}(\zeta)\right|}{\left\|R_{n}\right\|_{C(E)}}=\max _{q_{n} \in \mathscr{R}\left(A_{n}\right)} \frac{\left|q_{n}^{\prime}(\zeta)\right|}{\left\|q_{n}\right\|_{C(E)}} .
$$

Тогда существуют $\alpha$ и $\beta$ такие, что

$$
R_{n}(x)=\frac{\alpha x^{n}+\beta x^{n-1}+s_{n-2}(x)}{\prod_{l=1}^{n}\left(1-a_{l} x\right)},
$$

әде $s_{n-2}(x) / \prod_{l=1}^{n}\left(1-a_{l} x\right)$ есть наилучшая чебышевская аппроксимация функиии $\left(\alpha x^{n}+\beta x^{n-1}\right) / \prod_{l=1}^{n}\left(1-a_{l} x\right)$ на $E$.

Доказательство леммы дословно повторяет рассуждения, использованные при доказательстве теоремы 3 из [1] (для случая $A_{n}=\{0,0, \ldots, 0\}$ ).

ДокАЗАТЕЛЬСТво тЕоремЫ 1. Сначала предположим, что $R_{n} \in \mathscr{R}_{2, n}$ (т.е. $a_{i} \neq a_{j}$, $i \neq j$ ) и все нули $R_{n}$ удовлетворяют неравенству

$$
x<\min _{1 \leqslant l \leqslant n} \operatorname{Re} \frac{1}{a_{l}} .
$$

Пусть $E=[-1, a] \cup[b, 1]$ и $\tau \in E$. Пусть $R_{n} \in \mathscr{R}_{2, n}$ удовлетворяет равенству

$$
\left|R_{n}^{\prime}(\tau)\right|=\max _{\substack{q_{n} \in \mathscr{R}_{2, n} \\ q_{n} \neq 0}} \frac{\left|q_{n}^{\prime}(\zeta)\right|}{\left\|q_{n}\right\|_{C(E)}}
$$

и $\left\|R_{n}\right\|_{C(E)}=1$. Тогда нетрудно показать, как это было сделано в доказательстве теоремы 3 из [1], что все нули $R_{n}$ лежат на $E$, быть может за исключением $\lambda_{1} \in(a, b)$ и $\lambda_{2}>1$ или $\lambda_{2}<-1$. Предположим, что $\lambda_{2}>1$. Кроме того, без ограничения общности можно считать, что для $y \in\{-1, a, b, 1\}\left|R_{n}(y)\right|=1,\left|R_{n}^{\prime}(y)\right| \neq 0$ и для $y \in(a, b)$ $\left|R_{n}(y)\right|>1$. Будем считать также, что все $a_{l}$ различны. Таким образом, можно найти точки $a<\varepsilon_{1}<\delta_{1}<\lambda_{1}<\delta_{2}<\varepsilon_{2}<b$ и $1<\varepsilon_{3}<\delta_{3}<\lambda_{2}<\delta_{4}, \lambda_{2}<\min _{1 \leqslant l \leqslant n} \operatorname{Re}\left(1 / a_{l}\right)$ такие, что $R_{n}^{\prime}\left(\varepsilon_{i}\right)=0, i=1,2,3$, и $\left|R_{n}\left(\delta_{i}\right)\right|=1, i=1,2,3,4$. Используя неравенства

$$
\begin{gathered}
\left|\frac{\left(x-\varepsilon_{3}\right)^{2}}{\left(x-\delta_{3}\right)\left(x-\delta_{4}\right)}\right|<1 \quad \text { при } x \leqslant 1, \\
\left|\frac{\left(x-\varepsilon_{1}\right)^{2}}{\left(x-\delta_{1}\right)\left(x-\delta_{2}\right)}\right|<1 \quad \text { при } x \in(-1, a), \\
\left|\frac{1}{a_{l}}-\varepsilon_{2}\right|>\left|\frac{1}{a_{l}}-b\right|, \quad l=1, \ldots, n, \\
\left|x-\varepsilon_{2}\right|<|x-b|, \quad x \in(-1, a),
\end{gathered}
$$

получим оценку (1).

По непрерьвности требование отличия полюсов $1 / a_{l}$ друг от друга и от бесконечности можно снять. Тем самым, неравенство (1) из теоремы 1 будет доказано для $A_{n} \subset$ $\{z:|z-1 / 2|<1 / 2\}$ и рациональных функций с нулями на $\mathbb{C} \backslash\left[\min _{l} \operatorname{Re}\left(1 / a_{l}\right),+\infty\right)$. 
Перейдем к общему случаю. Очевидно, что множество $\left\{1 / a_{l}, l=\varkappa, \ldots, n\right\}$ можно считать расположенньм вне некоторой окружности с центром на действительной оси, проходящей через точку $x=1$ и содержащей внутри себя полуинтервал $[-1,1)$. Эта окружность пересекает действительную ось, помимо точки $x=1$, в точке $x=-\alpha$, $\alpha=\min _{\varkappa \leqslant l \leqslant n}\left|a_{l}\right|^{-1}$. Применение отображения

$$
w=\frac{z\left(1-a^{\prime} a\right)+a^{\prime}-a}{z\left(a^{\prime}-a\right)+1-a^{\prime} a}
$$

где $a^{\prime}=(1+\alpha a) /(\alpha+a)$, сводит доказательство $(1)$ к рассмотренному случаю. Поскольку при этом преобразовании внешность круга $|z|=\alpha$ перейдет в $\{w: \operatorname{Re} w>$ $\left.\left(1+\alpha^{2}\right) /(2 \alpha)\right\}$, неравенство (1) полностью доказано. Неравенство (2) доказьвается аналогично.

ЗАмечАниЕ 3. Теорема 1 асимптотически точна на рассматриваемом классе строк обратных величин полюсов.

Действительно, если все $a_{l}$ равны между собой, $a_{l}=\alpha_{n}, l=1, \ldots, n$, то условие регулярности строки $A_{n}$ превратится в условие $\rho_{l}=-p K / n, l=1, \ldots, n$, где

$$
\operatorname{sn}^{2} \rho_{l}=\frac{(1-a)\left(1+\alpha_{n}\right)}{2\left(1-a \alpha_{n}\right)} .
$$

Полагая теперь $p=n-1$, определим $\alpha_{n}$ из равенства

$$
\frac{(1-a)\left(1+\alpha_{n}\right)}{2\left(1-a \alpha_{n}\right)}=\operatorname{sn}^{2} \frac{(n-1) K}{n} .
$$

Так как правая часть этого равенства при $n \rightarrow \infty$ стремится к 1 , то и левая часть стремится к 1 , т.е. $\alpha_{n} \rightarrow 1$. Тогда производная дроби Чебьшева-Маркова, построенной по соответствующей строке $A_{n}$, будет равна (по теореме 2 ) величине

$$
\frac{n \sqrt{M_{n}^{2}-R_{n}^{2}\left(A_{n}, E, x\right)}}{\sqrt{\left(1-x^{2}\right)(x-a)(x-b)}} \frac{\sqrt{\left(1-\alpha_{n}^{2}\right)\left(1-\alpha_{n} a\right)\left(1-\alpha_{n} b\right)}}{1-\alpha_{n} x} \frac{x-c_{n}}{1-\alpha_{n} c_{n}}
$$

где

$$
c_{n}=\frac{\left(1-\alpha_{n} a\right) \Theta^{\prime}\left(\rho_{n-1}\right) \sqrt{(1-a)(1+b)}-a \Theta\left(\rho_{n-1}\right) \sqrt{\left(1-\alpha_{n}^{2}\right)\left(1-\alpha_{n} a\right)\left(1-\alpha_{n} b\right)}}{\alpha_{n}\left(1-\alpha_{n} a\right) \Theta^{\prime}\left(\rho_{n-1}\right) \sqrt{(1-a)(1+b)}-\Theta\left(\rho_{n-1}\right) \sqrt{\left(1-\alpha_{n}^{2}\right)\left(1-\alpha_{n} a\right)\left(1-\alpha_{n} b\right)}} .
$$

Поскольку

$$
\frac{\Theta^{\prime}(K)}{\Theta(K)} \sqrt{(1-a)(1+b)}=0
$$

и $\rho_{n-1} \rightarrow K$ при $n \rightarrow \infty$, для выбранной $A_{n}$ имеем

$$
\begin{aligned}
R_{n}^{\prime}\left(A_{n}, E, x\right)= & \frac{n \sqrt{M_{n}^{2}-R_{n}^{2}\left(A_{n}, E, x\right)}}{\sqrt{\left(1-x^{2}\right)(x-a)(x-b)}} \\
& \times\left(\frac{(x-a) \sqrt{\left(1-\alpha_{n}^{2}\right)\left(1-\alpha_{n} b\right)\left(1-\alpha_{n} a\right)}}{\left(1-\alpha_{n} x\right)\left(1-\alpha_{n} a\right)}+o(1)\right) .
\end{aligned}
$$

Именно в этом смысле и подразумевается асимптотическая точность неравенства (1). Асимптотическая точность неравенства (2) проверяется аналогично.

Автор благодарен А. И. Аптекареву за обсуждение результатов работы. 


\section{СПИСОК ЦИТИРОВАННОЙ ЛИТЕРАТУРЫ}

[1] Borwein P. B. Markov's and Bernstein's inequalities on disjoint intervals // Canad. J. Math. 1981. V. 33. № 1. P. 201-209.

[2] Ахиезер Н. И., Левин Б. Я. Обобщение неравенства С. Н. Бернштейна для производных от цельх функций // Исследования по современным проблемам теории функций комплексного переменного / ред. А.И. Маркушевич. М.: Физматгиз, 1960. С. 111-165.

[3] Виденский В.С. Некоторые оценки производных от рациональных дробей // Изв. АН CССР. Сер. матем. 1962. Т. 26. №3. C. 415-426.

[4] Borwein P. B., Erdelyi T., Zhang J. Chebyshev polynomials and Markov-Bernstein type inequalities for rational spaces // J. London Math. Soc. (2). 1994. V. 50. P. 501-519.

[5] Русак В. Н. Рациональные функции как аппарат приближения. Минск: Изд. БГУ, 1979.

[6] Пекарский А. А. Оценки производной интеграла типа Коши с мероморфной плотностью и их приложения // Матем. заметки. 1982. Т. 31. № 3. С. 389-402.

[7] Li X., Mohapatra R. N., Rodriguez R. S. Bernstein-type inequalities for rational functions with prescribed poles // J. London Math. Soc. (2). 1995. V. 51. P. 523-531.

[8] Данченко В.И. О разделении особенностей мероморфных функций // Матем. сб. 1984. T. 125. № 2. C. 181-198.

[9] Lorentz G. G., Golitschek M.V., Makovoz Y. Constructive Approximation. Advanced Problems. Berlin-Heidelberg: Springer, 1996.

[10] Rahman Q. I., Schmeisser G. Les inégalités de Markoff et de Bernstein. Montréal: Presses Univ. Montréal, 1983.

[11] Лукашов А. Л. О задаче Чебьшева-Маркова на двух отрезках // Деп. ВИНИТИ №6615-89. М.: ВИНИТИ, 1989.

[12] Achyeser N. I. Über einige Funktionen, welche in zwei gegebenen Intervallen am wenigsten von Null abweichen. I // Изв. АН СССР. Отд. матем. и естеств. наук. 1932. Т. 9. С. 1163-1202.

[13] Levy R. Generalized rational function in finite intervals using Zolotarev functions // IEEE Trans. Microwave Theory Tech. 1970. V. 18. № 12. P. 1052-1064.

[14] Уиттекер Э. Т., Ватсон Дж. Н. Курс современного анализа. Трансцендентные функции. Т. 2. М.: Физматлит, 1963.

[15] Peherstorfer F. Elliptic orthogonal and extremal polynomials // Proc. London Math. Soc. (3). 1995. V. 70. P. 605-624. 